\title{
1 Pre-attentive processing of neutral and emotional sounds in congenital amusia
}

4 Agathe Pralus ${ }^{1,2}$, Marie Gomot ${ }^{3}$, Jackson Graves ${ }^{1,4}$, Fanny Cholvy ${ }^{1,2}$, Lesly Fornoni ${ }^{1,2}$, Barbara

$5 \quad$ Tillmann $^{1,2 *}$, Anne Caclin ${ }^{1,2 *}$

$6 \quad{ }^{1}$ Lyon Neuroscience Research Center; CNRS, UMR5292; INSERM, U1028; Lyon, F-69000,

7 France

$8 \quad{ }^{2}$ University Lyon 1, Lyon, F-69000, France

$9 \quad{ }^{3}$ UMR 1253, INSERM, Université de Tours, Tours, France

$10{ }^{4}$ Department of Psychology, University of Minnesota, Minneapolis

$12 *$ Equally contributing authors.

13 Corresponding author: agathe.pralus@ inserm.fr CRNL, 95 Bd Pinel 69675 Bron Cedex, France

14 Declarations of interest: none

15

16 


\section{Abstract}

Congenital amusia is a life-long deficit of musical processing. This deficit can extend to the processing of language and in particular, emotional prosody. In a previous behavioral study, we revealed that while amusic individuals had difficulties in explicitly recognizing emotions for short vowels, they rated the emotional intensity of these same vowels as did their matched control participants. This finding led to the hypothesis that congenital amusics might be impaired for explicit emotional prosody recognition, but not for its implicit processing. With the aim to investigate amusics' automatic processing of prosody, the present study measured electroencephalography (EEG) when participants listened passively to vowels presented within an oddball paradigm. Emotionally neutral vowel served as the standard and either emotional (anger and sadness) or neutral vowels as deviants. Evoked potentials were compared between participants with congenital amusia and control participants matched in age, education, and musical training. The MMN was rather preserved for all deviants in amusia, whereas an earlier negative component was found decreased in amplitude in amusics compared to controls for the neutral and sadness deviants. For the most salient deviant (anger), the P3a was decreased in amplitude for amusics compared to controls. These results showed some preserved automatic detection of emotional deviance in amusia despite an early deficit to process subtle acoustic changes. In addition, the automatic attentional shift in response to salient deviants at later processing stages was reduced in amusics in comparison to the controls. In the three ERPs related to the deviance, between-group differences were larger over bilateral prefrontal areas, previously shown to display functional impairments in congenital amusia. Our present study thus provides further understanding of the dichotomy between implicit and explicit processing 41 in congenital amusia, in particular for vocal stimuli with emotional content. 


\section{Introduction}

Congenital amusia, also known as tone-deafness, is a life-long deficit of music processing. This

\& Vuvan, 2017), with potentially genetic origins (Peretz et al., 2007). Individuals with congenital amusia show no hearing impairments or brain lesions that could explain their deficit. They are usually unable to sing in tune or detect an out-of-key note (see Peretz, 2016; Tillmann et al., 2015 for reviews). Several studies have revealed a specific pitch processing deficit in congenital amusia, with pitch perception tasks (Hyde \& Peretz, 2004; Peretz et al., 2003) and pitch memory tasks (Albouy et al., 2016; Graves et al., 2019; Tillmann, Lévêque, et al., 2016; Williamson \& Stewart, 2010). The pitch deficit was observed for non-musical material, such as isolated pitches or tone pairs (Albouy et al., 2016; Foxton et al., 2004; Peretz et al., 2009), as well as tone sequences or melodies (see Tillmann et al., 2015 for a review). As pitch processing is relevant beyond the musical domain, the investigation of congenital amusia has been extended to speech perception abilities. While some early studies did not report any deficit of speech processing in amusic individuals (Ayotte et al., 2002; Tillmann et al., 2009; Williamson \& Stewart, 2010), more recent studies have revealed specific impairments of speech contour perception and intonation recognition in congenital amusia (Jiang et al., 2010; Liu et al., 2015, 2017; Nan et al., 2016; Nguyen et al., 2009; Tillmann, Burnham, et al., 2011; Tillmann, Rusconi, et al., 2011).

As pitch is essential to process emotions both in speech and music, some studies have started to investigate emotional processing in congenital amusia. Regarding musical emotion perception, congenital amusics have demonstrated either a mild impairment or no impairment 
in recognition tasks (Gosselin et al., 2015; Lévêque et al., 2018; Marin et al., 2015), but have shown preserved intensity ratings of the emotions (Lévêque et al., 2018). Regarding emotion perception in speech, referred to as emotional prosody, congenital amusics have demonstrated a mild deficit of recognition (Lima et al., 2016; Pralus et al., 2019; Thompson et al., 2012), which was more pronounced for short vowels (with few acoustic cues) than long sentences (Lolli et al., 2015; Pralus et al., 2019). This recognition deficit was the largest for sadness stimuli (Pralus et al., 2019; Thompson et al., 2012), which tended to be confounded with neutral stimuli (Pralus et al., 2019). Interestingly, when congenital amusics were asked to rate the intensity of emotional prosody stimuli, they did not show any deficit compared to matched controls, even for vowels (Pralus et al., 2019). Intensity ratings of emotions can be given without precise categorical representation of the emotion or explicit labeling, suggesting some preserved implicit processing of emotions in amusia (Lévêque et al., 2018; Pralus et al., 2019). For music material, preserved implicit processing of pitch in amusia has been reported, even though explicit processing has been shown to be disrupted (Lévêque et al., 2018; Omigie et al., 2013; Pralus et al., 2019; Tillmann et al., 2012, 2014; Tillmann, Lalitte, et al., 2016). For instance, congenital amusics were able to process pitch changes as well as pitch incongruity (Peretz et al., 2009; Zendel et al., 2015), even though they were unable to detect these changes or incongruities when explicitly asked to do so (Moreau et al., 2009; Omigie et al., 2012; Tillmann, Lévêque, et al., 2016). This recent research suggests congenital amusia to be a disorder of consciousness related to pitch representations (Albouy et al., 2016; Marin et al., 2015; Moreau et al., 2009, 2013; Omigie et al., 2013; Peretz, 2016; Peretz et al., 2009; Stewart, 2011; Tillmann, Lalitte, et al., 2016).

Aiming to further investigate this hypothesis, in the present study, we recorded several electrophysiological measures that have been previously used to explore implicit processes in the typical and the pathological brain. One target measure, which reflects pre-attentional 
processes in the brain, is the well-studied Mismatch Negativity (MMN) (Näätänen et al., 2007;

Näätänen \& Alho, 1995). This negative ERP component emerges when a deviant event appears in a repetitive auditory sequence (referred to as the oddball paradigm). It is considered to be automatic as it can be recorded even when participants are actively engaged in another task (Näätänen et al., 2007; Näätänen \& Alho, 1995). The MMN signal is very robust, stable, and found in most control participants at the individual level (Chen et al., 2018; Kraus et al., 1992). It is generally reported to originate from supratemporal and frontal cortical regions (Näätänen et al., 2007; Näätänen \& Alho, 1995). An MMN can be induced not only by simple acoustic deviants, as classically studied (Näätänen et al., 2007; Peretz et al., 2005), but also by emotional deviant events (Goydke et al., 2004). For emotional prosodic material, such as vowels, an MMN can be induced by an emotional deviant, compared to a neutral standard (Carminati et al., 2018; Charpentier et al., 2018). This emotional MMN occurs generally at a shorter latency and is larger than for neutral deviant (Schirmer et al., 2005, 2016).

For non-emotional material, amusics' automatic brain response to acoustic changes has been studied in passive listening paradigms with pitch tone deviants or tone-language stimuli (Fakche et al., 2018; Moreau et al., 2009, 2013; Nan et al., 2016; Omigie et al., 2013; Zhang \& Shao, 2018). Using pitch change passive paradigms, amusics' early change-related evoked potentials, such as the MMN, were decreased in amplitude in comparison to controls for small pitch changes (Fakche et al., 2018; Moreau et al., 2009, 2013). When the pitch change was large enough (200 cents), the MMN seemed to be preserved in amusics (Moreau et al., 2009, 2013). Omigie et al. (2013) used real melodies to investigate amusics' and controls' brain responses as a function of the degree of expectedness of the notes (Omigie et al., 2013). The results revealed that with increased unexpectedness the early negativity (in the N1 latency range) increased for controls, but not for amusics. It suggests a deficit in the processing of musical structures at early processing stages, in keeping with the results of Albouy et al. (2013) in an 
117 active short-term memory task for melodies. When tone-language stimuli were used, amusics

118 did not demonstrate any decrease of MMN in response to lexical tones (Nan et al., 2016; Zhang

$119 \&$ Shao, 2018).

120 In active paradigms, similar result patterns regarding early brain response to different acoustic

121 changes in congenital amusia have been observed (Braun et al., 2008; Peretz et al., 2005, 2009).

122 For pitch change detection tasks, the MMN was decreased for amusics (compared to controls)

123 only for small pitch deviants (Peretz et al., 2005, 2009). The replacement of the correct tone

124 with an incorrect (out-of-tune) deviant tone at the end of familiar melodies revealed a decreased

125 early negativity in amusics compared to controls (Braun et al., 2008). For language material, in

126 particular intonation processing with statements and questions, the early negativity was

127 preserved in amusics, but the N2 response was decreased in response to incongruent pairs of

128 tones (Lu et al., 2015).

129 Some alterations in deviance detection have been observed also for later components, such as

130 the P3 (Braun et al., 2008; Lu et al., 2015; Moreau et al., 2009, 2013; Peretz et al., 2009; Zhang \& Shao,

131 2018). For pitch change detection tasks using tones, a decreased $\mathrm{P} 3$ was observed for amusics

132 (in comparisons to controls) only for small pitch changes (25 cents), but not otherwise (Braun

133 et al., 2008; Moreau et al., 2013). For lexical tone changes, smaller P3a and P3b were observed

134 in amusics compared to controls for small lexical tone changes (high rising vs. low rising tone)

135 (Zhang \& Shao, 2018).

136 Overall, some results have shown decreased early electrophysiological markers related to pitch

137 deviance detection in congenital amusia, mostly for small pitch changes, and sometime together

138 with a reduction of the subsequent P3a. However, the pattern of automatic pitch processing in

139 speech and music in congenital amusia still needs further investigation. 
140 Our previous behavioral study investigating emotional prosody in congenital amusia has

141 suggested preserved implicit prosody processing (Pralus et al., 2019). With the aim to further

142 investigate amusics' automatic processing of prosody, the present study measured

143 electroencephalography (EEG) when participants listened passively to vowels presented within

144 an oddball paradigm. Emotionally neutral vowel served as the standard and either emotional

145 (anger and sadness) or neutral vowels as deviants. Evoked potentials were compared between

146 participants with congenital amusia and control participants matched in age, education, and

147 musical training. Emotional deviants (anger and sadness) and neutral deviant were chosen from

148 the material of our previous study (Pralus et al., 2019) aiming for similar F0 difference

149 compared to the neutral standard. Anger was the best recognized emotion by amusics whereas

150 sadness was not well recognized and often confused with neutrality. Hence, these two emotional

151 deviants had different patterns of recognition in the two participant groups, while intensity

152 ratings were similar across groups for these stimuli. We hypothesized that early automatic

153 processing of emotion deviancy will be impaired in amusics compared to controls, with

154 potentially different responses to neutral and emotional deviancy in these two groups. 


\section{Material and Methods}

157

158

\section{Participants}

Nineteen amusic participants and twenty-one control participants matched for gender, age, laterality, education, and musical training (as defined by years of instruction of an instrument) at the group level were included in the study (Table 1). They all gave written informed consent to participate in the experiment. Prior to the main experiment, all participants were tested with a subjective audiometry, the Montreal Battery of Evaluation of Amusia (Peretz et al., 2003) to diagnose amusia, and a Pitch Discrimination Threshold (PDT) test (Tillmann et al., 2009). A participant was considered amusic if he/she had a global MBEA score below 23 (maximum score $=30$ ) and/or a MBEA pitch score (average of the first three subtests of the MBEA) inferior to 22 (maximum score $=30$ ). All control participants had a global MBEA score above 24.5 and a MBEA pitch score above 23.3 (see Table 1). All participants had normal hearing (hearing loss inferior to $30 \mathrm{~dB}$ at any frequency in both ears). Study procedures were approved by a national ethics committee. Participants provided written informed consent prior to the experiment and were paid for their participation.

\section{Stimuli}

Four vowels /a/ were selected from a larger material set, all produced with female voices (Charpentier et al., 2018), and used in a previous behavioral study with amusic $(\mathrm{N}=18)$ and control (N=18) participants (Pralus et al., 2019). All stimuli lasted $400 \mathrm{~ms}$ and were equalized in RMS amplitude. The stimuli were selected based on their recognition scores in the behavioral task (Pralus et al., 2019, see Table 2) as follows: the neutral deviant and standard were equally well recognized by all participants; the anger deviant was selected as an easy deviant (equally well-recognized by both groups); the sadness deviant was selected as a difficult deviant for amusics. We added the constraint that all stimuli should be similar in pitch and should have received similar intensity ratings (for emotional stimuli) (see Table 2 for details). Acoustic 
181 parameters (pitch mean, spectral flux mean, brightness mean, roughness mean, inharmonicity

182 mean, and attack time) of the stimuli were computed with the MIR toolbox (Lartillot \&

183 Toiviainen, 2007); Table 2). Each parameter (except Attack Time) was computed with a

184 temporal frame of $50 \mathrm{~ms}$ by default. We then computed the average of each parameter across

185 time (see Table 2).

186

187

188

189

\section{Procedure}

The experiment took place in a sound-attenuated room. Participants watched a silent movie with subtitles, they were told to not pay attention to the sounds played over headphones. The recording session lasted 45 minutes.

\section{EEG recordings and ERP measurements}

The entire experimental paradigm was composed of three oddball blocks, each with one type of deviant (Neutral, Sadness, Anger) and one block with equiprobable stimuli. For each oddball block, 700 standards and 140 deviants were played. Two consecutive deviants were separated by at least three standards. During the equiprobable block, each of the 4 stimuli were played equally often (144 times each, 576 stimuli in total), with no more than two repetitions of the same stimulus in a row. The stimulus onset asynchrony (SOA) was always $700 \mathrm{~ms}$.

EEG was recorded using 31 active electrodes (BrainAmp/Acticap, Brain Products, Germany) with a nose reference, with a sampling frequency of $1000 \mathrm{~Hz}$ (bandwidth $0.016-1000 \mathrm{~Hz}$ ). Eye movements were recorded with an electrode under the left eye (offline re-referenced to Fp1). ELAN software was used for EEG signal processing (Aguera et al., 2011). Band-stop filters centered around $50 \mathrm{~Hz}$ and $150 \mathrm{~Hz}$ were applied to the EEG signal to remove power line artifacts. Independent Component Analysis was performed on the EEG signal to remove artifacts due to eye movements and heartbeat (Delorme \& Makeig, 2004). Averaging was done for each deviant and standard separately, in the three oddball blocks and the equiprobable block. Standards 
occurring after a deviant were not averaged. Averaging was done on a 700ms time-window

206 (from -200 ms to $500 \mathrm{~ms}$ around stimulus onset). Trials with peak-to-peak amplitude variation

corrected by subtracting the average of the signal in the $100 \mathrm{~ms}$ before the stimulus. The

210 difference wave for each type of deviant (Neutral, Sadness, Anger) was obtained by subtracting

211 the response to the deviant from the response to the standard in the same block of the oddball paradigm $^{1}$. Grand-averaged curves were obtained for both groups (Amusics and Controls). The emergence of deviance-related ERPs (MMN and P3a in particular) was assessed with the

214 comparison of deviant and standard ERPs using a nonparametric cluster-based permutation 215 analysis (1000 permutations), in each group, for each of the three deviants. A first threshold of $216 \mathrm{p}<0.05$ was used for permutation-based paired t-tests for each sample. Clusters were labeled as significant for $\mathrm{p}<0.05$ at the end of the permutations, controlling for multiple comparisons in space ( 31 electrodes) and time. Based on the union of these emergence tests in both groups, two 219 or three time windows of interest were selected for each emotion. For neutral deviant, three 220 time-windows were selected: 67-130ms, 130-205ms, 225-310ms. For sadness deviant, three time-windows were selected: 77-140ms, 140-200ms, 220-295ms. For anger deviant, two timewindows were selected: $113-205 \mathrm{~ms}, 217-299 \mathrm{~ms}$. The first window corresponds to an early

\footnotetext{
${ }^{1}$ The equiprobable stimuli could not be used as the reference stimulus to compute difference ERPs, as in the equiprobable block, the anger sound (and to a lesser extent the neutral deviant sound) elicited a negativity compared to the other equiprobable sounds in the latency range of the MMN, suggesting that MMNs were elicited within this sequence (see Figure S1).
} 


\section{Statistical analysis}

226 Based on the emergence tests described above, a set of fronto-central electrodes was selected

227 for the main analysis. Average amplitude for electrode sites along the antero-posterior axis (four 228 levels) and for the two sides (pre-frontal=Fp1, Fp2, frontal=F3, F4, fronto-central=FC1, FC2, 229 central=C3, C4, odd numbers correspond to electrodes on the left side, even numbers on the 230 right side) were computed for each participant, for each type of deviant, in each of the time 231 windows of interest. For each emotion (neutral, sadness, anger) and for each time-window 232 (early negativity, MMN, P3, except for anger for which there was no early negativity), a 233 Bayesian repeated-measures Analysis of Variance (ANOVA) was performed with group 234 (Amusics, Controls) as a between-subjects factor, and localization (Fp, F, FC and C) and side (left, right) as within-subject factors ${ }^{2}$.

We report Bayes Factor $(\mathrm{BF})$ as a relative measure of evidence. To interpret the strength of 237 evidence (according to Lee \& Wagenmakers, 2014), we considered a BF under three as weak 238 evidence, a BF between three and 10 as positive evidence, a BF between 10 and 100 as strong evidence and a $\mathrm{BF}$ higher than 100 as a decisive evidence. $\mathrm{BF}_{10}$ indicates the evidence of $\mathrm{H} 1$ (a

240 given model) compared to $\mathrm{H} 0$ (the null model), and BF inclusion indicates the evidence of one 241 effect over all models. As no post-hoc tests with correction for multiple comparison have as yet 242 been developed for Bayesian statistics (Wagenmakers et al., 2017, 2018), we used t-tests with 243 Holm-Bonferroni correction for multiple comparisons.

\section{Data availability}

245 Raw data were generated at Lyon Neuroscience Research Center (France). Derived data supporting the findings of this study are available from the corresponding author upon request.

\footnotetext{
${ }^{2}$ We also computed Bayesian ANOVA on the amplitude at midline electrodes ( $\left.\mathrm{Fz}, \mathrm{Cz}, \mathrm{Pz}\right)$ for each time-window for each emotion. See Supplementary analysis for details.
} 


\section{Results}

248 Based on the emergence tests, three deviance-related ERPs were identified in the difference

249 curves (Figures 1-3): (1) an early negativity was observed, namely a negative fronto-central 250 deflection in a time-window of $\sim 70-140 \mathrm{~ms}$ after the stimulus onset; (2) the MMN was identified 251 as the negative fronto-central deflection in a time-window of $\sim 140-200 \mathrm{~ms}$ after the stimulus onset, associated with the typical polarity inversion at the mastoids; (3) the P3a was identified as the positive fronto-central deflection in a time-window of $\sim 220-300 \mathrm{~ms}$ after the stimulus onset, with a polarity inversion at the mastoids. See Figures 1-3A for averaged curves over fronto-central sites and Figures 1-3B for topographies. For the Anger deviant difference curve, only two emergence windows were retrieved, corresponding to the MMN and the P3a. For precise emergence windows for each emotion and each group, see Figures 1-3C. Overall, the morphology of deviance-related responses was slightly different across emotions. In particular, there were differences in the latencies of the ERPs across emotions, these latencies were similar between groups.

Only averaged curves with neutral and sadness deviants showed an early negativity on frontocentral electrodes (Figures 1-2B), which is at the latency of the N1: For the neutral deviant, the emergence was between 67 and 130ms, whereas it was later for the sadness deviant, between 77 and 140ms. As habituation of the N1 was visible on standards in the oddball blocks (Figure S2), this early negativity might mainly reflect the different degrees of habituation of the N1 between standards and deviants. This effect was possibly less pronounced for the anger deviant, which had a slower attack time (see Table 2), and resulted in later auditory ERPs (see in 268 particular the delay in the P50 with respect to the other stimuli in the equiprobable block, Figure 269 S1). At the MMN latency, a typical ERP was observed for the three types of deviants, emerging at different latencies for each emotion: 130-205ms for neutrality, 140-200ms for sadness, 113- 
272 the P3a latency, the three deviance-related ERPs had an emerging peak with different latencies

273 for each emotion: 225-310ms for neutrality, 220-295 for sadness, 217-299ms for anger.

274 Based on these observations, and in particular, that ERPs and their latencies were not identical 275 in the three emotions, the main analyses were performed separately by emotion and by 276 component.

Response to a neutral deviant (Figure 1)

After comparison to the null model, the best model showing decisive evidence was the model with the main effects of Localization, Group, and the interaction between the two

293 observed in controls (all pcorr>0.39).

295 After comparison to the null model, the best model showing decisive evidence was the model 
the model with the main effects of Localization and Group (BF10=2.52e+14), 7.1 times better than the model with the main effects of Localization and Side $(B F 10=4.66 e+13)$, and 9.3 times better than the model with the main effects of Localization, Group, and Side (BF10=3.59e+13). confirmed by a decisive specific effect of Localization (BFinclusion=6.43e+13), and no other specific effects (BFinclusion<0.31). According to post-hoc tests, amplitude at Fp and C sites was smaller than amplitudes at F, FC (all pcorr $<0.001$ ). The Group effect emerging in the second best model showed that amusics tended to have a smaller MMN than controls.

\section{P3a}

After comparison to the null model, the best model showing decisive evidence was the model with the main effects of Localization and Side $(B F 10=2.05 e+10)$. This model was 1.9 times better than the model with the main effects of Localization, Side, and Group (BF10=1.07e+10), and 2.3 times better than the model with the main effects of Localization, Side, Group, and the better than the other models $(\mathrm{BF} 10<2.05 \mathrm{e}+9)$. This was confirmed by a decisive specific effect

312 of Localization (BFinclusion=1.57e+9), a positive effect of Side (BFinclusion=6.01), and no 313 other specific effects (BFinclusion<0.59). Amplitudes were larger over left side than right side.

314 The Group effect emerging in the second best model showed that amusics tended to have a 315 bigger P3a than controls. According to post-hoc tests, amplitudes at Fp and C sites were smaller 316 than amplitudes at F and FC (all pcorr $<0.001)$.

Response to a sadness deviant (Figure 2)

\section{Early negativity}

319 After comparison to the null model, the best model showing decisive evidence was the model with the main effects of Localization, Group and the interaction between the two 
Localization $(\mathrm{BF} 10=1.68 \mathrm{e}+9)$, and 1.43 times better than the model with the main effects of

323 Localization and Group $(\mathrm{BF} 10=1.22 \mathrm{e}+9)$. The best model was at least 8.3 times better than the

324 other models (BF10<2.1e+8). This was confirmed by a decisive specific effect of Localization

325 (BFinclusion=9.36e+8), and no other specific effects (BFinclusion<1.37). Amusics had a

326 smaller early negativity than controls. According to post-hoc tests, amplitude at Fp sites was

327 smaller than amplitudes at F, FC (both pcorr $<0.001$ ), amplitudes at $\mathrm{F}$ and $\mathrm{C}$ were smaller than

328 amplitude at FC (both pcorr $<0.007$ ). Specifically, amusics had smaller amplitude at Fp

329 compared to amplitudes at F, FC, C (all pcorr $<0.004)$, whereas controls had smaller amplitude

330 at Fp compared only to FC (pcorr $=0.021$ ).

\section{MMN}

After comparison to the null model, the best model showing decisive evidence was the model with the main effect of Localization $(B F 10=2.79 e+10)$. This model was 1.64 times better than the model with the main effects of Localization and Group (BF10=1.7e+10), and 3.79 times better than the model with the main effects of Localization and Side $(\mathrm{BF} 10=7.36 \mathrm{e}+9)$. The best model was at least 6.2 times better than the other models $(\mathrm{BF} 10<4.52 \mathrm{e}+9)$. This was confirmed by a decisive specific effect of Localization (BFinclusion=1.2e+10), and no other specific effects (BFinclusion $<0.29$ ). The Group effect emerging in the second best model showed that the amusics tended to have a smaller MMN than controls. According to post-hoc tests, amplitudes at Fp and $\mathrm{C}$ were smaller than amplitudes at FC and F (all pcorr $<0.004$ ).

\section{P3a}

After comparison to the null model, the best model showing decisive evidence was the model with the main effect of Localization $(B F 10=6.25 \mathrm{e}+15)$. This model was 1.63 times better than the model with the main effects of Localization and Group (BF10=3.83e+15), and 7.98 times better than the model with the main effects of Localization and Side $(B F 10=7.83 e+14)$. The best model was at least 14 times better than the other models $(\mathrm{BF} 10<4.44 \mathrm{e}+14)$. This was 


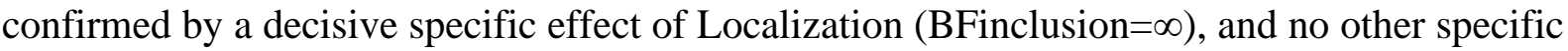

348 effects (BFinclusion<0.23). The Group effect emerging in the second best model showed that

349 amusics tended to have a smaller P3a than controls. According to post-hoc, amplitude at Fp was

350 smaller than amplitudes at F, C and FC (all pcorr $<0.015$ ), amplitude at $\mathrm{C}$ was smaller than

351 amplitudes at F and FC (both pcorr $<0.041$ ).

Response to an anger deviant (Figure 3)

After comparison to the null model, the best model showing decisive evidence was the model with the main effect of Localization $(B F 10=5.99 \mathrm{e}+14)$. This model was 1.74 times better than the model with the main effects of Localization and Group (BF10=3.45e+14), and 4.68 times better than the model with the main effects of Localization and Group and the interaction between the two $(\mathrm{BF} 10=1.28 \mathrm{e}+14)$. The best model was at least 8.14 times better than the other models $(\mathrm{BF} 10<7.36 \mathrm{e}+14)$. This was confirmed by a decisive specific effect of Localization 360 (BFinclusion=9.46e+13), and no other specific effects (BFinclusion<0.3). The Group effect emerging in the second best model showed that amusics tended to have a smaller MMN than

$\mathrm{F}$ (all pcorr $<0.006$ ), amplitude at $\mathrm{C}$ and $\mathrm{F}$ were smaller than amplitude at $\mathrm{FC}$ (both pcorr $<0.035$ ).

After comparison to the null model, the best model showing decisive evidence was the model with the main effects of Localization and Group and the interaction between the two $(B F 10=3.25 e+16)$. This model was 4.6 times better than the model with the main effects of 368 Localization, Side, Group, and the interaction between Localization and Group $369(\mathrm{BF} 10=7.02 \mathrm{e}+15)$, and 6.1 times better than the model with the main effects of Localization and Group $(\mathrm{BF} 10=5.3 \mathrm{e}+15)$. The best model was at least 11.9 times better than the other models 371 (BF10<2.95e+15). This was confirmed by a decisive specific effect of Localization 


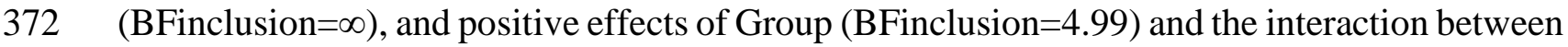

373 Localization and Group (BFinclusion=9.23), and no other specific effects (BFinclusion<0.09).

374 According to post-hoc tests, amplitude at Fp was smaller than amplitudes at C, FC and F (all

375 pcorr $<0.017$ ), amplitudes at $\mathrm{C}$ and $\mathrm{F}$ were smaller than amplitude at $\mathrm{FC}$ (both pcorr $<0.001$ ),

376 amplitude at $\mathrm{C}$ was smaller than amplitude at $\mathrm{F}$ (pcorr=0.032). Amusics had a significantly

377 smaller P3 compared to Controls. This group difference was especially observed at Fp sites

378 (pcorr=0.081). Specifically, amusics had smaller amplitude at Fp compared to amplitudes at F,

379 FC, C (all pcorr <0.001), whereas controls had smaller amplitude at Fp compared only to FC

$380 \quad($ pcorr $=0.002)$.

381 Comparisons between deviants

382 To investigate potential differences across emotions, we ran a Bayesian ANOVA with the

383 additional within-subjects factor Emotion for each evoked potential.

384

385

\section{Early negativity}

This analysis included only the neutral and sadness deviants. After comparison to the null model, the best model showing decisive evidence was the model with the main effects of Localization and Emotion $(\mathrm{BF} 10=3.2 \mathrm{e}+10)$. This model was 1.4 times better than the model with the main effects of Localization, Emotion, Group and the interaction between Localization and Group $(\mathrm{BF} 10=2.3 \mathrm{e}+10)$, and 1.6 times better than the model with the main effects of Localization, Emotion and Group $(\mathrm{BF} 10=2.05 \mathrm{e}+10)$. The best model was at least 4.1 times better than the other models $(\mathrm{BF} 10<7.8 \mathrm{e}+9)$. This was confirmed by a decisive specific effect of Localization (BFinclusion=6.17e+8), a small positive effect of Emotion (BFinclusion=2.68) and no other specific effects (BFinclusion<0.51). According to post-hoc tests, the amplitude at Fp sites was smaller than amplitudes at F, FC and C (all pcorr $<0.012$ ), amplitude at FC was larger than amplitudes at $\mathrm{F}$ and $\mathrm{C}($ all pcorr $<0.001)$. The early negativity for sadness was smaller than the one for neutrality. Amusics tended to have a smaller early negativity than controls. 
Specifically, amusics tended to have smaller amplitude at Fp compared to amplitudes at F, FC,

C (all pcorr $<0.001$ ), whereas controls tended to have smaller amplitudes at Fp compared to amplitudes at FC only (pcorr=0.019).

400

401

402

403

404

405

406

407

408

409

\section{2. $M M N$}

After comparison to the null model, the best model showing decisive evidence was the model with the main effects of Localization and Emotion $(\mathrm{BF} 10=1.6 \mathrm{e}+18)$. This model was 2.2 times better than the model with the main effects of Localization, Emotion and Group $(\mathrm{BF} 10=7.15 \mathrm{e}+17)$, and 13.3 times better than the model with the main effects of Localization, Emotion and Side $(\mathrm{BF} 10=1.2 \mathrm{e}+17)$. The best model was at least 18.8 times better than the other models $(\mathrm{BF} 10<8.49 \mathrm{e}+16)$. This was confirmed by a decisive specific effect of Localization $($ BFinclusion=2.82e+13) and Emotion (BFinclusion=782), and no other specific effects (BFinclusion $<0.071$ ). The Group effect emerging in the second best model showed that amusics tended to have a smaller MMN than controls. According to post-hoc tests, amplitude at Fp and C sites was smaller than amplitudes at F, FC (all pcorr <0.001), amplitude at Fp was smaller than amplitude at $\mathrm{C}$ (pcorr=0.029). The $\mathrm{MMN}$ tended to be smaller for sadness compared to anger (pcorr $=0.2)$.

\section{P3a}

After comparison to the null model, the best model showing decisive evidence was the model with the main effects of Localization, Emotion, Side, and Group, and the interaction between Emotion and Localization, between Emotion and Side, between Side and Localization, between Emotion and Group, and the triple interaction between Emotion, Localization and Side $(B F 10=9.85 e+82)$. This model was 16.2 times better than the model with the main effects of Localization, Emotion, Side and Group and the interaction between Emotion and Localization, between Emotion and Side, between Side and Localization, between Emotion and Group, between Side and Group, and the triple interaction between Emotion, Localization and Side 
$(\mathrm{BF} 10=6.08 \mathrm{e}+81)$, and 78 times better than the model with the main effects Localization,

423 Emotion, Side and Group and the interaction between Emotion and Localization, between

424 Emotion and Side, between Side and Localization, between Emotion and Group, between

425 Localization and Group, and the triple interaction between Emotion, Localization and Side

$426(\mathrm{BF} 10=1.26 \mathrm{e}+81)$. The best model was at least 99 times better than the other models

427 (BF10<9.9e+80). This was confirmed by a decisive specific effect of Localization

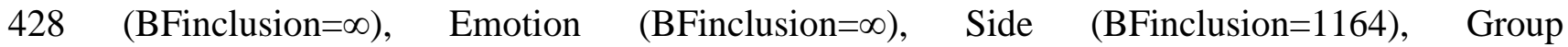

429 (BFinclusion=6949), the interaction between Emotion and Localization

430 (BFinclusion=1.24e+10), the interaction between Emotion and Side (BFinclusion=664), the

431 interaction between Emotion and Group (BFinclusion=33214), the interaction between

432 Localization and Side (BFinclusion=676) and the interaction between Emotion, Localization

433 and Side (BFinclusion=4740), and no other specific effects (BFinclusion<0.07). Amusics had

434 a smaller P3a compared to Controls. According to post-hoc tests, amplitude at Fp was smaller

435 than amplitudes at $\mathrm{C}, \mathrm{FC}$ and $\mathrm{F}$ (all pcorr $<0.016$ ), amplitudes at $\mathrm{C}$ and $\mathrm{F}$ were smaller than

436 amplitude at $\mathrm{FC}$ (both pcorr <0.016), amplitude at $\mathrm{C}$ was smaller than amplitude at $\mathrm{F}$

437 (pcorr $<0.001$ ). The P3a was larger for anger compared to sadness and neutrality (both

438 pcorr $<0.046$ ), and larger for sadness compared to neutrality (pcorr $<0.001)$. More specifically,

439 amplitudes for neutrality was smaller than amplitude for anger and sadness at C, F and FC (all

440 pcorr $<0.002$ ), amplitude for neutrality was smaller than amplitude for anger at Fp

441 (pcorr=0.002). Neutrality had smaller amplitude than had sadness and anger for both left and

442 right sides (all pcorr $<0.001$ ). Amplitudes were larger over left side than right side. This was

443 driven by a smaller amplitude at $\mathrm{C}$ for right side compared to left side (pcorr=0.098). This

444 difference was driven in particular by the difference between the two groups for anger

445 (pcorr=0.1). In amusics, neutrality had smaller amplitude than had anger (pcorr=0.025), 
446 whereas in controls, neutrality had smaller amplitude than had anger and sadness (both pcorr $<0.004)$.

The analyses of components amplitude at midline electrodes $(\mathrm{Fz}, \mathrm{Cz}, \mathrm{Pz})$ are reported in the supplementary material. Only limited group effects were observed in these analyses, in keeping with the results reported above which reveal that between-group differences were mostly observed at prefrontal sites, for which we did not have a midline electrode in our 32-electrode montage. These results at midline electrodes further emphasize that the early negativity peaking at $\mathrm{Fz}$ and $\mathrm{Cz}$ was slightly more central than the MMN, which peaked at Fz. This is in agreement with the hypothesis that the early negativity included N1 refractoriness effects.

\section{Discussion}

Using an oddball paradigm with emotional prosody stimuli, we revealed the automatic brain responses of congenital amusic individuals compared to matched control participants for neutral and emotional verbal sounds. Based on previous behavioral and ERP results, we expected a decreased early automatic processing of deviancy in amusics compared to controls, with potentially different responses to neutral and emotional deviancy in these two groups. Amusics

462 had reduced automatic processing of a neutral deviant compared to controls, with a diminished 463 early negativity at the latency of N1 and a slightly reduced MMN. Similarly, the early 464 processing of emotional stimuli (reflected by the early negativity at the latency of N1) was decreased in amusics compared to controls, yet with only slightly reduced emotional MMNs. The later P3a observed in response to a salient emotional deviant (anger) was strongly decreased

467 in amusics compared to controls. These results suggest a differential processing of neutrality 468 and emotions, with impaired pre-attentive processing of both neutral and emotional sounds in 
470

471

472 473 deviances.

474

475

476

477

478

479

480

stages associated with high-level cognitive processes (around $300 \mathrm{~ms}$ ). The rather preserved MMN in between these altered processing stages suggest that change detection mechanisms can operate on degraded initial sound representations, at least in the case of large enough sound

Even if congenital amusia was first described to be music-specific (Ayotte et al., 2002; Peretz et al., 2003), recent evidence suggest that the pitch deficit in congenital amusia could also extend to speech material, even though to a lesser extent (Nguyen et al., 2009; Tillmann, Burnham, et al., 2011; Tillmann, Rusconi, et al., 2011; Zhang et al., 2017). In relation with the present study, congenital amusia is not only a music perception deficit but also a language processing deficit, in particular for non-verbal auditory cues such as emotional prosody (Lolli et al., 2015; Nguyen et al., 2009; Patel et al., 2008; Pralus et al., 2019; Thompson et al., 2012).

\section{Impaired early encoding of auditory stimuli in congenital amusia}

A smaller early negativity was observed in amusics compared to controls for neutral and sadness deviants. It points to amusics' increased difficulties to automatically process the deviants at early processing stages. This early negativity seems to correspond to N1 adaptation effects as the adaptation observed here occurs in the latency range of the N1, with a slightly different topography than the subsequent MMN.

In agreement with previous research (Albouy et al., 2013; Omigie et al., 2013), our results thus reveal an early deficit of auditory encoding in the amusics' brain. This early processing seems to be particularly less efficient for neutral stimuli, but can also be altered for emotional stimuli, as revealed by the results with the sadness deviant. Interestingly, taken together, the results suggest a general decrease of the early negativity in congenital amusia, observed both in the processing of pitch sequences (Albouy et al., 2013; Omigie et al., 2013) and in oddball contexts (current results). As suggested by stimulus-specific adaptation research (Carbajal \& Malmierca, 
2018; Malmierca et al., 2014; Pérez-González \& Malmierca, 2014), a precise representation of

495

496

497

498

499

500

501

502

503

504

505

506

507

508

509

510

511

512

the standard is necessary to elicit a strong N1 when the deviant is presented. However, if the representation of the standard is not precise, as in congenital amusics, the N1 elicited by the deviant remains similar to the N1 elicited by the standard, as revealed here using an oddball paradigm.

Interestingly, similar pitch deviance was used with the three types of deviant (the smaller pitch deviance was for the sadness deviancy). Even though we tried to match the acoustic differences between the three types of deviant as closely as possible, other acoustic features than pitch differentiated between the three emotions. These variations of acoustic parameters could explain at least in part the pattern of evoked responses in the two groups. As roughness and inharmonicity were higher for the anger deviant compared to sadness and neutrality, it could have helped amusics to correctly process this anger deviant and recognize it behaviorally (Pralus et al., 2019). Indeed, previous reports suggest that amusics' emotional judgments are based largely on roughness and tempo rather than harmonicity cues, which are mostly used by controls (Gosselin et al., 2015; Lévêque et al., 2018; Marin et al., 2015). Moreover, the anger deviant was characterized by a longer attack time, in particular when compared to the neutral standard. This could explain why the pattern of the first evoked potentials in response to this deviant was different compared to the two other emotions, with no early negativity at the latency of N1. However, this specific pattern of responses was similar in the two groups.

\section{Preserved automatic change detection and implicit processes in amusia}

To investigate automatic change detection processes in congenital amusia, we studied the MMN evoked by the three deviants. As expected (Carminati et al., 2018; Charpentier et al., 2018), a MMN was induced by both emotional and neutral deviants, compared to a neutral standard in both groups. The MMN was larger for emotional deviants than for the neutral deviant (Schirmer et al., 2005, 2016). 
No clear deficit of the MMN for the neutral or emotional deviants was observed in amusics compared to controls, suggesting at least a partially preserved automatic processing of emotional prosody in amusics, as previously shown with behavioral data (Lima et al., 2016; research on automatic pitch processing in congenital amusia demonstrating only a small deficit of the congenital amusics' MMN for small pitch changes in tone sequences (Fakche et al., 2018;

525 Moreau et al., 2009, 2013; Nan et al., 2016; Omigie et al., 2013; Zhang \& Shao, 2018). It suggests that, despite an impaired early processing of the deviant, congenital amusics' brain is still able to automatically detect the change. However, even if it was not significant, we did observe a small decrease of the MMN to the neutral deviant in amusics, suggesting that this implicit knowledge in amusics' brain might not be fully sufficient in some cases to allow the change detection mechanisms underlying the MMN to produce as large error signals as in controls. It is widely admitted that a correct sensory memory representation of the standard is needed to elicit an MMN (Näätänen et al., 2005). This would suggest that in congenital amusic participants, this memory representation is not as accurate as in controls. Thus, these results

534 would contribute to the understanding of the deficit in congenital amusia as previously 535 demonstrated with short-term memory tasks (Albouy et al., 2013, 2016; Fakche et al., 2018; Graves et al., 2019; Tillmann et al., 2009; Williamson \& Stewart, 2010).

In combination with the analysis of the early negativity, these results show that acoustic

538 sensitivity is impaired in congenital amusia, and do not seem to depend on emotional content 539 of the stimulus. However, the more cognitive and memory-related comparison reflected by the 540 MMN (Maess et al., 2007) seems to be less impaired in congenital amusia. In particular, it 541 appears that this component would be only minimally impacted in congenital amusia when an 542 emotional component is present in the stimulus. Such preserved automatic cortical processing 543 steps could be the basis of the preserved implicit processes observed behaviorally in musical 
544 and emotional judgements (Lévêque et al., 2018; Pfeuty \& Peretz, 2010; Pralus et al., 2019;

545 Stewart, 2011; Tillmann et al., 2007; Tillmann, Lalitte, et al., 2016).

\section{Decreased awareness of emotional stimuli in congenital amusia}

547 To further investigate the potential deficit of awareness in congenital amusia for emotional 548 stimuli, we analyzed the P3a in response to the three types of deviants. This ERP was larger for

549 the emotional deviants, especially for anger, but was still detectable for the two deviants in the 550 two participant groups. For the anger deviant, which elicited the largest P3a in controls, the P3a 551 was strongly decreased in amusics compared to controls. A reduced P3a in amusics was 552 previously shown with lexical tones (Zhang \& Shao, 2018) and using tasks with small pitch 553 changes in tone sequences (Braun et al., 2008; Moreau et al., 2009, 2013). The decreased P3a 554 relates to an awareness deficit suggested in congenital amusia (Peretz et al., 2009), in particular 555 for emotional stimuli (Lévêque et al., 2018; Pralus et al., 2019). Specifically, P3a is considered 556 to reflect automatic attentional orientation toward a salient deviant (Escera et al., 1998; Polich $557 \&$ Criado, 2006). Thus, congenital amusics would have a deficit to process unexpected novel 558 sounds. However, amusics were still able to perform the recognition task for the anger deviant. 559 These results suggest that when the automatic preattentional processes of the amusics reach a 560 sufficient level (a sizeable MMN and a detectable P3a), they can perform the recognition task, 561 despite this deficit at these late processing stages.

\section{Brain networks involved in emotional prosody perception in congenital amusia}

563 The group differences were mostly visible on bilateral pre-frontal electrodes. Interestingly, in 564 congenital amusia, frontal regions were found to be altered (Albouy et al., 2013, 2019; Hyde et 565 al., 2006, 2007, 2011). In particular, decreased gray and white matter volume of the inferior 566 frontal cortices was observed in congenital amusia (Albouy et al., 2013, 2019; Hyde et al., 2006, 567 2007, 2011). As these regions are involved in emotional prosody processing (Frühholz et al., 568 2012; Liu et al., 2015), it could have been expected that amusics would have a deficit to perceive 
emotional prosody. However, our results with the MMN suggest a partial preservation of these circuits to automatically detect emotional prosody in congenital amusics. These results are in

571 line with previous reports showing that the perception of emotional prosody does not only

572 involve a fronto-temporal network, but also extend to other regions, such as probably the 573 amygdala that detects salience and meaningful information (Frühholz et al., 2016), which would 574 be preserved in congenital amusia. Further research using brain imaging (with fMRI for example) should investigate the brain networks involved in emotional perception in congenital 576 amusia.

\section{Conclusion}

578 Our present findings shed new light on different aspects of automatic sound processing in 579 congenital amusia, in particular for speech material and its emotional features. The observed 580 impairments might lead to difficulties to process speech correctly in some situations. For 581 instance, in degraded conditions such as hearing in noise, challenging conditions for speech 582 comprehension (Liu et al., 2015; Oxenham, 2008, 2012; Tang et al., 2018), amusics could have 583 more difficulties to understand the speaker's emotions and intentions (Mcdonald \& Stewart, 584 2008; Omigie et al., 2012). Moreover, this study gives further insight about the dissociation of 585 implicit and explicit processing in congenital amusia (Lévêque et al., 2018; Omigie et al., 2013; 586 Pralus et al., 2019; Tillmann et al., 2012, 2014; Tillmann, Lalitte, et al., 2016). It reveals the 587 overall pattern of emotional perception in congenital amusia, from the first steps of cortical 588 processing (Albouy et al., 2013; Omigie et al., 2013) to the late processing stages (P300, 589 Moreau et al., 2009, 2013; Peretz et al., 2009), via the intermediate stage of change detection 590 reflected by the MMN. This relatively preserved MMN might relate to preserved implicit 591 processing in congenital amusia for music and emotional prosody stimuli. 


\section{Fundings}

593 This work was conducted in the framework of the LabEx CeLyA ("Centre Lyonnais 594 d'Acoustique", ANR-10-LABX-0060) and of the LabEx Cortex ("Construction, Cognitive 595 Function and Rehabilitation of the Cortex", ANR-11-LABX-0042) of Université de Lyon, 596 within the program "Investissements d'avenir" (ANR-16-IDEX-0005) operated by the French 597 National Research Agency (ANR).

\section{References}

600 Aguera, P.-E., Jerbi, K., Caclin, A., \& Bertrand, O. (2011). ELAN: a software package for 601 analysis and visualization of MEG, EEG, and LFP signals. Computational Intelligence and Neuroscience, 2011, 158970. https://doi.org/10.1155/2011/158970

604

605

Albouy, P., Caclin, A., Norman-Haignere, S. V., Lévêque, Y., Peretz, I., Tillmann, B., \& Zatorre, R. J. (2019). Decoding Task-Related Functional Brain Imaging Data to Identify

Albouy, P., Cousineau, M., Caclin, A., Tillmann, B., \& Peretz, I. (2016). Impaired encoding of 608 rapid pitch information underlies perception and memory deficits in congenital amusia. Scientific Reports, 6, 18861. https://doi.org/10.1038/srep18861 Delpuech, C., Bertrand, O., Caclin, A., \& Tillmann, B. (2013). Impaired pitch perception and memory in congenital amusia: the deficit starts in the auditory cortex. Brain: A Journal of Neurology, 136(Pt 5), 1639-1661. https://doi.org/10.1093/brain/awt082 
615 Ayotte, J., Peretz, I., \& Hyde, K. (2002). Congenital amusiaA group study of adults afflicted

616

617

618

620

621 with a music-specific disorder. Brain, 125(2), 238-251. https://doi.org/10.1093/brain/awf028

Braun, A., McArdle, J., Jones, J., Nechaev, V., Zalewski, C., Brewer, C., \& Drayna, D. (2008). Tune Deafness: Processing Melodic Errors Outside of Conscious Awareness as Reflected by Components of the Auditory ERP. PLoS ONE, 3(6). https://doi.org/10.1371/journal.pone.0002349

Carbajal, G. V., \& Malmierca, M. S. (2018). The Neuronal Basis of Predictive Coding Along the Auditory Pathway: From the Subcortical Roots to Cortical Deviance Detection. Trends in Hearing, 22. https://doi.org/10.1177/2331216518784822

Carminati, M., Fiori-Duharcourt, N., \& Isel, F. (2018). Neurophysiological differentiation between preattentive and attentive processing of emotional expressions on French vowels. Biological Psychology, 132 $55-63$. https://doi.org/10.1016/j.biopsycho.2017.10.013

Charpentier, J., Kovarski, K., Roux, S., Houy-Durand, E., Saby, A., Bonnet-Brilhault, F., Latinus, M., \& Gomot, M. (2018). Brain mechanisms involved in angry prosody change detection in school-age children and adults, revealed by electrophysiology. Cognitive, Affective \& Behavioral Neuroscience. https://doi.org/10.3758/s13415-018-0602-8

Chen, C., Chan, C.-W., \& Cheng, Y. (2018). Test-Retest Reliability of Mismatch Negativity (MMN) to Emotional Voices. Frontiers in Human Neuroscience, 12. https://doi.org/10.3389/fnhum.2018.00453

Delorme, A., \& Makeig, S. (2004). EEGLAB: an open source toolbox for analysis of singletrial EEG dynamics including independent component analysis. Journal of Neuroscience Methods, 134(1), 9-21. https://doi.org/10.1016/j.jneumeth.2003.10.009 
639 Escera, C., Alho, K., Winkler, I., \& Näätänen, R. (1998). Neural Mechanisms of Involuntary

640

641

642 Attention to Acoustic Novelty and Change. Journal of Cognitive Neuroscience, 10(5), 590-604. https://doi.org/10.1162/089892998562997

Fakche, C., Lecaignard, F., Lévêque, Y., Fornoni, L., Tillmann, B., \& Caclin, A. (2018). Impaired short-term memory for pitch in congenital amusia: a MEG/EEG study of Mismatch Negativity. Poster Presented at MMN2018: 8th Mis-match Neg-at-iv-ity Conference, Helsinki.

Foxton, J. M., Dean, J. L., Gee, R., Peretz, I., \& Griffiths, T. D. (2004). Characterization of deficits in pitch perception underlying 'tone deafness.' Brain, 127(4), 801-810. https://doi.org/10.1093/brain/awh105

Frühholz, S., Ceravolo, L., \& Grandjean, D. (2012). Specific Brain Networks during Explicit and Implicit Decoding of Emotional Prosody. Cerebral Cortex, 22(5), 1107-1117. https://doi.org/10.1093/cercor/bhr184

Frühholz, S., Trost, W., \& Kotz, S. A. (2016). The sound of emotions-Towards a unifying neural network perspective of affective sound processing. Neuroscience and Biobehavioral Reviews, 68, 96-110. https://doi.org/10.1016/j.neubiorev.2016.05.002

Gosselin, N., Paquette, S., \& Peretz, I. (2015). Sensitivity to musical emotions in congenital amusia. Cortex; a Journal Devoted to the Study of the Nervous System and Behavior, 71, 171-182. https://doi.org/10.1016/j.cortex.2015.06.022

Goydke, K. N., Altenmüller, E., Möller, J., \& Münte, T. F. (2004). Changes in emotional tone and instrumental timbre are reflected by the mismatch negativity. Cognitive Brain Research, 21(3), 351-359. https://doi.org/10.1016/j.cogbrainres.2004.06.009

Graves, J. E., Pralus, A., Fornoni, L., Oxenham, A. J., Caclin, A., \& Tillmann, B. (2019). Shortand long-term memory for pitch and non-pitch contours: Insights from congenital 
663

664

665

666

667

668

669

670

671

672

673

674

675

676

677

678

679

680

681

682

683

684

685

686

amusia.

Brain

and

Cognition,

136,

103614. https://doi.org/10.1016/j.bandc.2019.103614

Hyde, K. L., Lerch, J. P., Zatorre, R. J., Griffiths, T. D., Evans, A. C., \& Peretz, I. (2007). Cortical Thickness in Congenital Amusia: When Less Is Better Than More. Journal of Neuroscience, 27(47), 13028-13032. https://doi.org/10.1523/JNEUROSCI.303907.2007

Hyde, K. L., \& Peretz, I. (2004). Brains That Are out of Tune but in Time. Psychological Science, 15(5), 356-360. https://doi.org/10.1111/j.0956-7976.2004.00683.x

Hyde, K. L., Zatorre, R. J., Griffiths, T. D., Lerch, J. P., \& Peretz, I. (2006). Morphometry of the amusic brain: a two-site study. Brain: A Journal of Neurology, 129(Pt 10), 25622570. https://doi.org/10.1093/brain/awl204

Hyde, K. L., Zatorre, R. J., \& Peretz, I. (2011). Functional MRI evidence of an abnormal neural network for pitch processing in congenital amusia. Cerebral Cortex (New York, N.Y.: 1991), 21(2), 292-299. https://doi.org/10.1093/cercor/bhq094

Jiang, C., Hamm, J. P., Lim, V. K., Kirk, I. J., \& Yang, Y. (2010). Processing melodic contour and speech intonation in congenital amusics with Mandarin Chinese. Neuropsychologia, 48(9), 2630-2639. https://doi.org/10.1016/j.neuropsychologia.2010.05.009

Kraus, N., McGee, T., Sharma, A., Carrell, T., \& Nicol, T. (1992). Mismatch negativity eventrelated potential elicited by speech stimuli. Ear and Hearing, 13(3), 158-164.

Lartillot, O., \& Toiviainen, P. (2007). Mir in Matlab (ii): A Toolbox for Musical Feature Extraction from Audio. Austrian Computer Society.

Lee, M. D., \& Wagenmakers, E.-J. (2014). Bayesian Cognitive Modeling: A Practical Course. Cambridge Core - Psychology Research Methods and Statistics. https://doi.org/10.1017/CBO9781139087759 
Lévêque, Y., Teyssier, P., Bouchet, P., Bigand, E., Caclin, A., \& Tillmann, B. (2018). Musical emotions in congenital amusia: Impaired recognition, but preserved emotional intensity. Neuropsychology. https://doi.org/10.1037/neu0000461

Lima, C. F., Brancatisano, O., Fancourt, A., Müllensiefen, D., Scott, S. K., Warren, J. D., \& Stewart, L. (2016). Impaired socio-emotional processing in a developmental music disorder. Scientific Reports, 6. https://doi.org/10.1038/srep34911

Liu, F., Jiang, C., Francart, T., Chan, A. H. D., \& Wong, P. C. M. (2017). Perceptual learning of pitch direction in congenital amusia: evidence from Chinese speakers. Music Perception, 34(3), 335-351.

Liu, F., Jiang, C., Wang, B., Xu, Y., \& Patel, A. D. (2015). A music perception disorder (congenital amusia) influences speech comprehension. Neuropsychologia, 66, 111-118. https://doi.org/10.1016/j.neuropsychologia.2014.11.001

Lolli, S. L., Lewenstein, A. D., Basurto, J., Winnik, S., \& Loui, P. (2015). Sound frequency affects speech emotion perception: results from congenital amusia. Frontiers in Psychology, 6, 1340. https://doi.org/10.3389/fpsyg.2015.01340

Lu, X., Ho, H. T., Liu, F., Wu, D., \& Thompson, W. F. (2015). Intonation processing deficits of emotional words among Mandarin Chinese speakers with congenital amusia: an ERP study. Frontiers in Psychology, 6. https://doi.org/10.3389/fpsyg.2015.00385

Maess, B., Jacobsen, T., Schröger, E., \& Friederici, A. D. (2007). Localizing pre-attentive auditory memory-based comparison: Magnetic mismatch negativity to pitch change. NeuroImage, 37(2), 561-571. https://doi.org/10.1016/j.neuroimage.2007.05.040

Malmierca, M. S., Sanchez-Vives, M. V., Escera, C., \& Bendixen, A. (2014). Neuronal adaptation, novelty detection and regularity encoding in audition. Frontiers in Systems Neuroscience, 8. https://doi.org/10.3389/fnsys.2014.00111 
Marin, M. M., Thompson, W. F., Gingras, B., \& Stewart, L. (2015). Affective evaluation of simultaneous tone combinations in congenital amusia. Neuropsychologia, 78, 207-220. https://doi.org/10.1016/j.neuropsychologia.2015.10.004

Mcdonald, C., \& Stewart, L. (2008). Uses and functions of music in congenital amusia. Music Perception 25(4):345. https://doi.org/10.1525/MP.2008.25.4.345

Moreau, P., Jolicoeur, P., \& Peretz, I. (2009). Automatic brain responses to pitch changes in congenital amusia. Annals of the New York Academy of Sciences, 1169, 191-194. https://doi.org/10.1111/j.1749-6632.2009.04775.x

Moreau, P., Jolicœur, P., \& Peretz, I. (2013). Pitch discrimination without awareness in congenital amusia: evidence from event-related potentials. Brain and Cognition, 81(3), 337-344. https://doi.org/10.1016/j.bandc.2013.01.004

Näätänen, R., \& Alho, K. (1995). Generators of electrical and magnetic mismatch responses in humans. Brain Topography, 7(4), 315-320. https://doi.org/10.1007/BF01195257

Näätänen, R., Jacobsen, T., \& Winkler, I. (2005). Memory-based or afferent processes in mismatch negativity (MMN): a review of the evidence. Psychophysiology, 42(1), 2532.

Näätänen, R., Paavilainen, P., Rinne, T., \& Alho, K. (2007). The mismatch negativity (MMN) in basic research of central auditory processing: A review. Clinical Neurophysiology, 118(12), 2544-2590. https://doi.org/10.1016/j.clinph.2007.04.026

Nan, Y., Huang, W. T., Wang, W. J., Liu, C., \& Dong, Q. (2016). Subgroup differences in the lexical tone mismatch negativity (MMN) among Mandarin speakers with congenital amusia. Biological Psychology, 113 $59-67$. https://doi.org/10.1016/j.biopsycho.2015.11.010 
734 Nguyen, S., Tillmann, B., Gosselin, N., \& Peretz, I. (2009). Tonal language processing in

735

736 congenital amusia. Annals of the New York Academy of Sciences, 1169, 490-493. https://doi.org/10.1111/j.1749-6632.2009.04855.x

Omigie, D., Müllensiefen, D., \& Stewart, L. (2012). The Experience of Music in Congenital Amusia. Music Perception: An Interdisciplinary Journal, 30(1), 1-18. https://doi.org/10.1525/mp.2012.30.1.1

Omigie, D., Pearce, M. T., Williamson, V. J., \& Stewart, L. (2013). Electrophysiological correlates of melodic processing in congenital amusia. Neuropsychologia, 51(9), 17491762. https://doi.org/10.1016/j.neuropsychologia.2013.05.010

Oxenham, A. J. (2008). Pitch Perception and Auditory Stream Segregation: Implications for Hearing Loss and Cochlear Implants. Trends in Amplification, 12(4), 316-331. https://doi.org/10.1177/1084713808325881

Oxenham, A. J. (2012). Pitch perception. The Journal of Neuroscience: The Official Journal of the Society for Neuroscience, 32(39), 13335-13338. https://doi.org/10.1523/JNEUROSCI.3815-12.2012

Patel, A. D., Wong, M., Foxton, J., Lochy, A., \& Peretz, I. (2008). Speech Intonation Perception Deficits in Musical Tone Deafness (congenital Amusia). Music Perception: An Interdisciplinary Journal, 25(4), 357-368. https://doi.org/10.1525/mp.2008.25.4.357

Peretz, I. (2016). Neurobiology of Congenital Amusia. Trends in Cognitive Sciences, 20(11), 857-867. https://doi.org/10.1016/j.tics.2016.09.002

Peretz, I., Brattico, E., Järvenpää, M., \& Tervaniemi, M. (2009). The amusic brain: in tune, out of key, and unaware. Brain: A Journal of Neurology, 132(Pt 5), 1277-1286. https://doi.org/10.1093/brain/awp055 
Peretz, I., Brattico, E., \& Tervaniemi, M. (2005). Abnormal electrical brain responses to pitch in congenital amusia. Annals of Neurology, 58(3), 478-482. https://doi.org/10.1002/ana.20606

760

761

762

763

764

765

766

767

768

769

770

771

772

773

774

775

776

777 Battery of Evaluation of Amusia. Annals of the New York Academy of Sciences, 999, $58-75$.

Peretz, I., Cummings, S., \& Dubé, M.-P. (2007). The Genetics of Congenital Amusia (Tone Deafness): A Family-Aggregation Study. American Journal of Human Genetics, 81(3), $582-588$.

Peretz, I., \& Vuvan, D. T. (2017). Prevalence of congenital amusia. European Journal of Human Genetics: EJHG, 25(5), 625-630. https://doi.org/10.1038/ejhg.2017.15

Pérez-González, D., \& Malmierca, M. S. (2014). Adaptation in the auditory system: an overview. Frontiers in Integrative Neuroscience,

8. https://doi.org/10.3389/fnint.2014.00019

Pfeuty, M., \& Peretz, I. (2010). Abnormal pitch--time interference in congenital amusia: evidence from an implicit test. Attention, Perception \& Psychophysics, 72(3), 763-774. https://doi.org/10.3758/APP.72.3.763

Polich, J., \& Criado, J. R. (2006). Neuropsychology and neuropharmacology of P3a and P3b. International Journal of Psychophysiology, 60(2), 172-185. https://doi.org/10.1016/j.ijpsycho.2005.12.012

Pralus, A., Fornoni, L., Bouet, R., Gomot, M., Bhatara, A., Tillmann, B., \& Caclin, A. (2019). Emotional prosody in congenital amusia: Impaired and spared processes. Neuropsychologia, 107234. https://doi.org/10.1016/j.neuropsychologia.2019.107234 
Schirmer, A., Escoffier, N., Cheng, X., Feng, Y., \& Penney, T. (2016). Detecting Temporal Change in Dynamic Sounds: On the Role of Stimulus Duration, Speed, and Emotion. Frontiers in Psychology, 6. https://doi.org/10.3389/fpsyg.2015.02055

783

784

Schirmer, A., Striano, T., \& Friederici, A. (2005). Sex differences in the preattentive processing of vocal emotional expressions. https://core.ac.uk/display/45977046

Stewart, L. (2011). Characterizing congenital amusia. Quarterly Journal of Experimental Psychology (2006), 64(4), 625-638. https://doi.org/10.1080/17470218.2011.552730

Tang, W., Wang, X.-J., Li, J.-Q., Liu, C., Dong, Q., \& Nan, Y. (2018). Vowel and tone recognition in quiet and in noise among Mandarin-speaking amusics. Hearing Research, 363, 62-69. https://doi.org/10.1016/j.heares.2018.03.004

Thompson, W. F., Marin, M. M., \& Stewart, L. (2012). Reduced sensitivity to emotional prosody in congenital amusia rekindles the musical protolanguage hypothesis. Proceedings of the National Academy of Sciences, 109(46), 19027-19032. https://doi.org/10.1073/pnas.1210344109

Tillmann, B., Albouy, P., \& Caclin, A. (2015). Congenital amusias. Handbook of Clinical Neurology, 129, 589-605. https://doi.org/10.1016/B978-0-444-62630-1.00033-0

Tillmann, B., Albouy, P., Caclin, A., \& Bigand, E. (2014). Musical familiarity in congenital amusia: evidence from a gating paradigm. Cortex; a Journal Devoted to the Study of the Nervous System and Behavior, 59, 84-94. https://doi.org/10.1016/j.cortex.2014.07.012

Tillmann, B., Burnham, D., Nguyen, S., Grimault, N., Gosselin, N., \& Peretz, I. (2011). Congenital Amusia (or Tone-Deafness) Interferes with Pitch Processing in Tone Languages. Frontiers in Psychology, 2, 120. https://doi.org/10.3389/fpsyg.2011.00120

Tillmann, B., Gosselin, N., Bigand, E., \& Peretz, I. (2012). Priming paradigm reveals harmonic structure processing in congenital amusia. Cortex; a Journal Devoted to the Study of the 
804

805

806

807

808

809

810

811

812

813

814

815

816

818

820

821

825

826

828

Nervous System and Behavior, 48(8), 1073-1078. https://doi.org/10.1016/j.cortex.2012.01.001

Tillmann, B., Lalitte, P., Albouy, P., Caclin, A., \& Bigand, E. (2016). Discrimination of tonal and atonal music in congenital amusia: The advantage of implicit tasks. Neuropsychologia, 85, 10-18. https://doi.org/10.1016/j.neuropsychologia.2016.02.027

Tillmann, B., Lévêque, Y., Fornoni, L., Albouy, P., \& Caclin, A. (2016). Impaired short-term memory for pitch in congenital amusia. Brain Research, 1640, Part B, 251-263. https://doi.org/10.1016/j.brainres.2015.10.035

Tillmann, B., Peretz, I., Bigand, E., \& Gosselin, N. (2007). Harmonic priming in an amusic patient: The power of implicit tasks. Cognitive Neuropsychology, 24(6), 603-622. https://doi.org/10.1080/02643290701609527

Tillmann, B., Rusconi, E., Traube, C., Butterworth, B., Umiltà, C., \& Peretz, I. (2011). Finegrained pitch processing of music and speech in congenital amusia. The Journal of the Acoustical Society of America, 130(6), 4089-4096. https://doi.org/10.1121/1.3658447

Tillmann, B., Schulze, K., \& Foxton, J. M. (2009). Congenital amusia: a short-term memory deficit for non-verbal, but not verbal sounds. Brain and Cognition, 71(3), 259-264. https://doi.org/10.1016/j.bandc.2009.08.003

Wagenmakers, E.-J., Love, J., Marsman, M., Jamil, T., Ly, A., Verhagen, J., Selker, R., Gronau, Q. F., Dropmann, D., Boutin, B., Meerhoff, F., Knight, P., Raj, A., Kesteren, E.-J. van, Doorn, J. van, Šmíra, M., Epskamp, S., Etz, A., Matzke, D., ... Morey, R. D. (2017). Bayesian inference for psychology. Part II: Example applications with JASP. Psychonomic Bulletin \& Review, 1-19. https://doi.org/10.3758/s13423-017-1323-7

Wagenmakers, E.-J., Marsman, M., Jamil, T., Ly, A., Verhagen, J., Love, J., Selker, R., Gronau, Q. F., Šmíra, M., Epskamp, S., Matzke, D., Rouder, J. N., \& Morey, R. D. (2018). Bayesian inference for psychology. Part I: Theoretical advantages and practical 
ramifications. Psychonomic Bulletin \& Review, 25(1), 35-57. https://doi.org/10.3758/s13423-017-1343-3

831 Williamson, V. J., \& Stewart, L. (2010). Memory for pitch in congenital amusia: beyond a finegrained pitch discrimination problem. Memory (Hove, England), 18(6), 657-669. https://doi.org/10.1080/09658211.2010.501339

Zendel, B. R., Lagrois, M.-É., Robitaille, N., \& Peretz, I. (2015). Attending to Pitch Information Inhibits Processing of Pitch Information: The Curious Case of Amusia. The Journal of Neuroscience, 35(9), 3815-3824. https://doi.org/10.1523/JNEUROSCI.3766-14.2015 tones in Cantonese-speaking congenital amusics. Scientific Reports, 8(1), 8420. https://doi.org/10.1038/s41598-018-26368-7

840 Zhang, C., Shao, J., \& Huang, X. (2017). Deficits of congenital amusia beyond pitch: Evidence from impaired categorical perception of vowels in Cantonese-speaking congenital amusics. PloS One, 12(8), e0183151. https://doi.org/10.1371/journal.pone.0183151 
bioRxiv preprint doi: https://doi.org/10.1101/2020.08.05.238204; this version posted August 5, 2020. The copyright holder for this preprint (which was not certified by peer review) is the author/funder, who has granted bioRxiv a license to display the preprint in perpetuity. It is made available under aCC-BY-NC-ND 4.0 International license.

A)

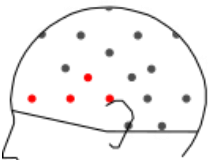

Left Fp

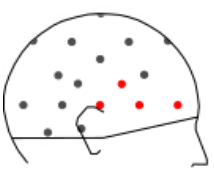

Right Fp

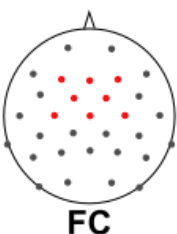

FC

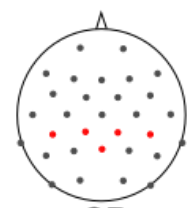

$\mathrm{CP}$

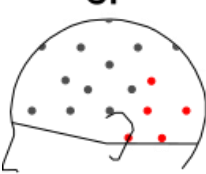

Left TP

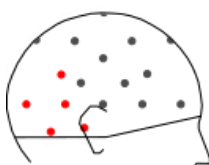

Right TP
AMUSICS

CONTROLS

B)

AMUSICS

CONTROLS
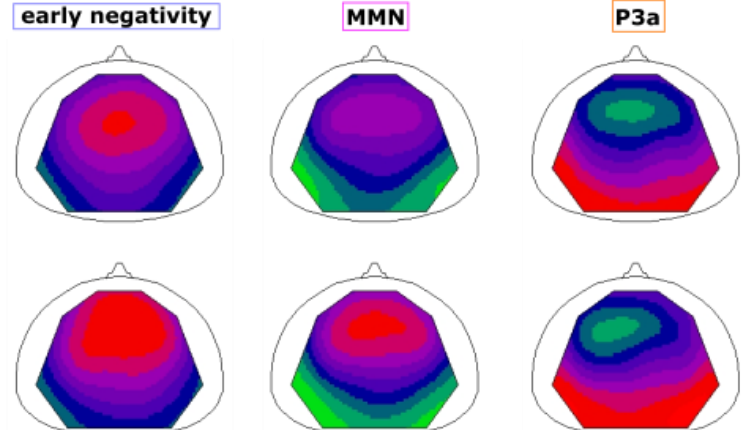

$+/-1.5 \mathrm{uV}$

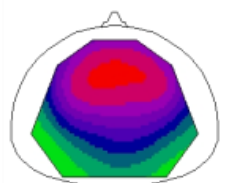

$+/-1.7$ uV

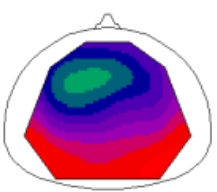

$+/-0.7 \mathrm{uV}$

C)

AMUSICS
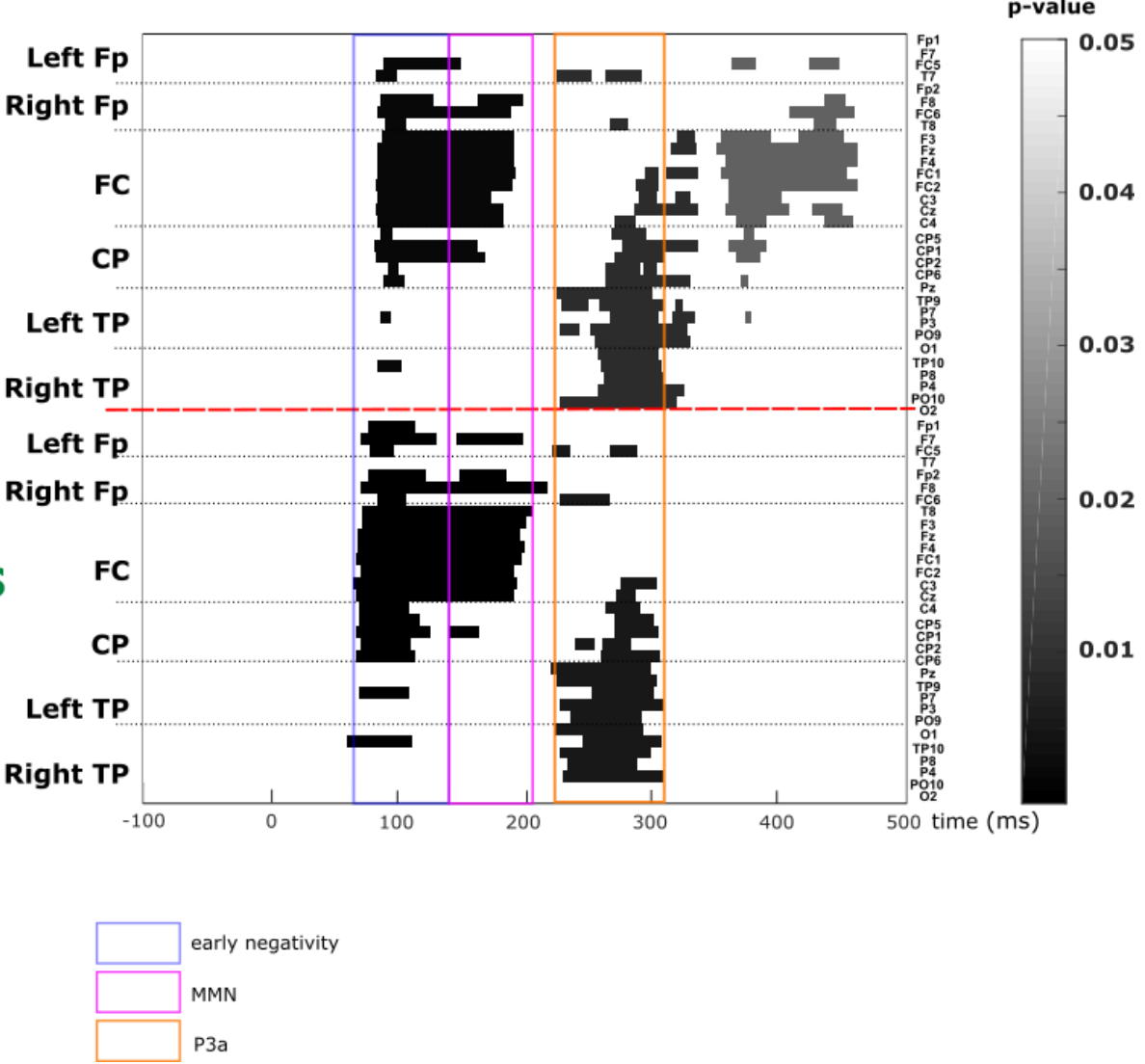

Figure 1: Evoked response to an emotionally neutral deviant in Amusics and Controls. A) Average curve of eight fronto-central electrodes (Fp1, Fp2, F3, F4, FC1, FC2, C3, C4) of the response to the neutral deviant minus the response to the neutral standard, for amusics and controls, negativity is up. B) Topographies for the three evoked potentials (early negativity, MMN and P3) over the emergence windows identified below, separately for amusics and controls. Amplitude scale is indicated for each ERP. C) Emergence of evoked responses for amusics and controls for each electrode, grouped by topography, emergence windows used for the analysis are in blue for early negativity $(67-130 \mathrm{~ms})$, pink for the MMN (130-205ms), orange for the P3a (225-310ms). Fp= pre-frontal, $\mathrm{FC}=$ fronto-central, $\mathrm{CP}=$ centro-parietal, $\mathrm{TP}=$ temporo-parietal. 
bioRxiv preprint doi: https://doi.org/10.1101/2020.08.05.238204; this version posted August 5, 2020. The copyright holder for this preprint (which was not certified by peer review) is the author/funder, who has granted bioRxiv a license to display the preprint in perpetuity. It is made available under aCC-BY-NC-ND 4.0 International license.

A)

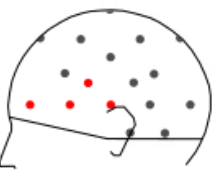

Left Fp

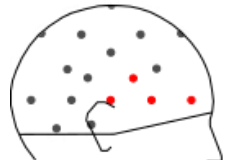

Right Fp

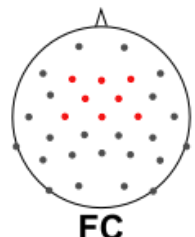

FC

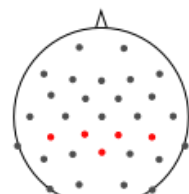

CP

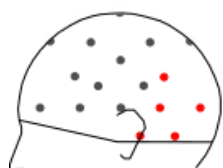

Left TP

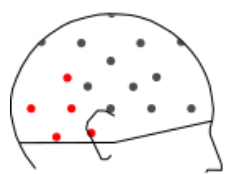

Right TP
AMUSICS

CONTROLS

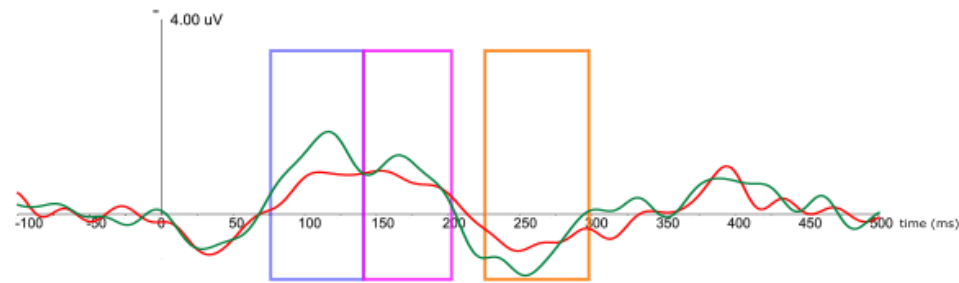

B)

AMUSICS
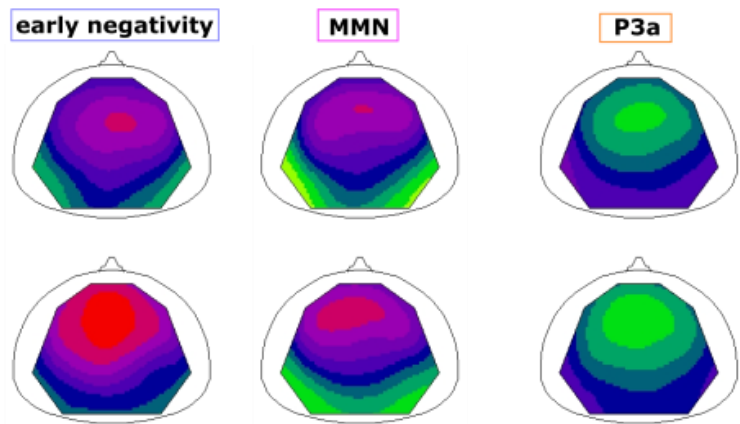

$+/-1.5$ uV

$+/-1.7 \mathrm{uV}$

$+/-1.7$ uV

C)
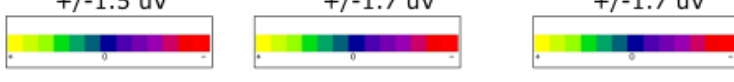

AMUSICS

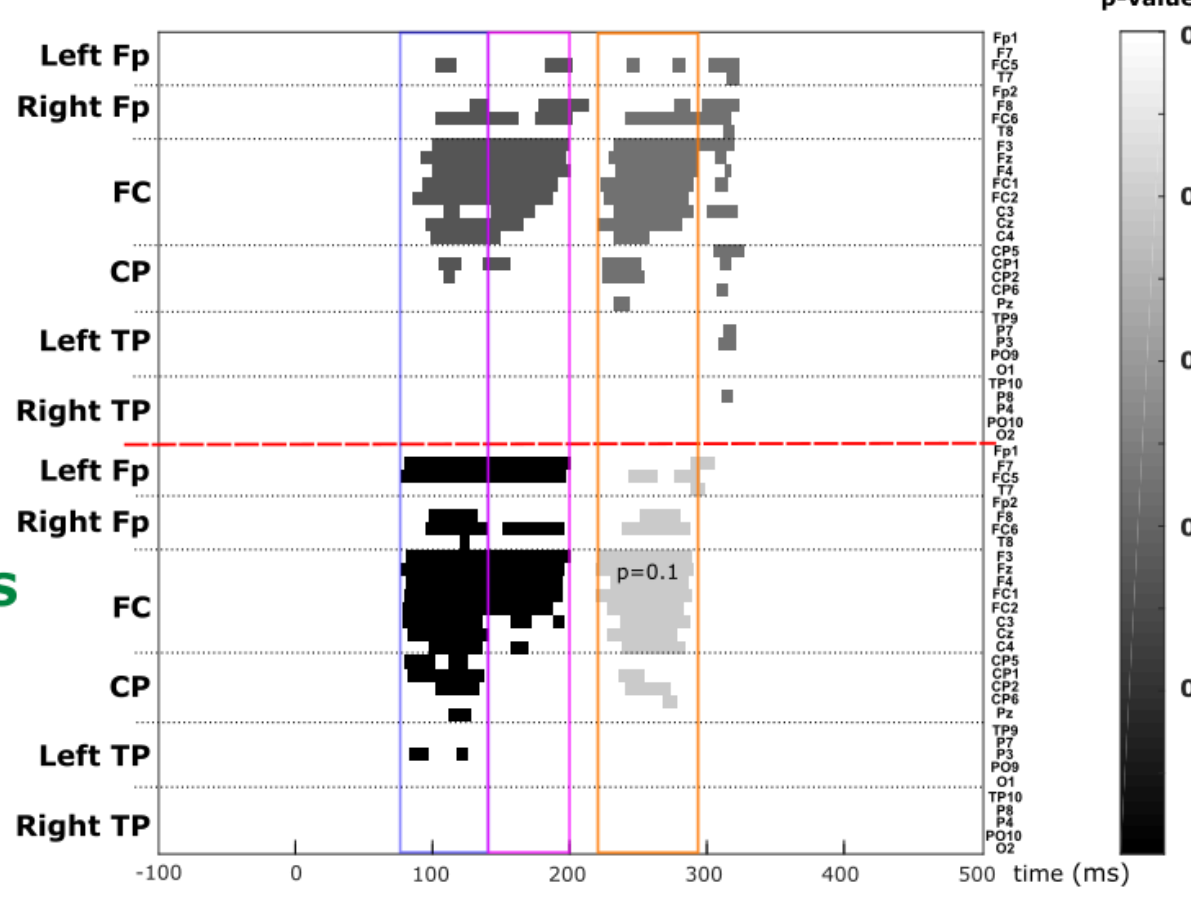

Figure 2: Evoked response to an emotional sadness deviant in Amusics and Controls. A) Average curve of eight fronto-central electrodes (Fp1, Fp2, F3, F4, FC1, FC2, C3, C4) of the response to the sadness deviant minus the response to the neutral standard, for amusics and controls, negativity is up. B) Topographies for the three evoked potentials (early negativity, MMN and P3) over the emergence windows identified below, separately for amusics and controls. Amplitude scale is indicated for each ERP. C) Emergence of evoked responses for amusics and controls for each electrode, grouped by topography, emergence windows used for the analysis are in blue for early negativity (77-140ms), pink for the MMN (140-200ms), orange for the P3a (220-295ms). The P3a only emerged in the amusic group at a pvalue of 0.05 for the permutation test, if the pvalue was set at 0.1 it also emerged in the control group. Fp= pre-frontal, $\mathrm{FC}=$ fronto-central, $\mathrm{CP}=$ centro-parietal, $\mathrm{TP}=$ temporo-parietal. 
bioRxiv preprint doi: https://doi.org/10.1101/2020.08.05.238204; this version posted August 5, 2020. The copyright holder for this preprint (which was not certified by peer review) is the author/funder, who has granted bioRxiv a license to display the preprint in perpetuity. It is made available under aCC-BY-NC-ND 4.0 International license.

A)

B)

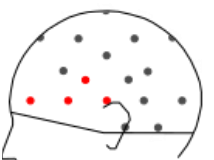

CONTROLS

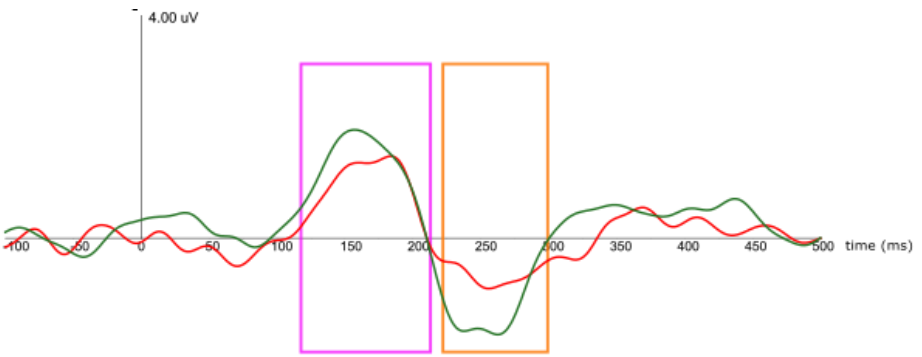

Left Fp

C)

AMUSICS
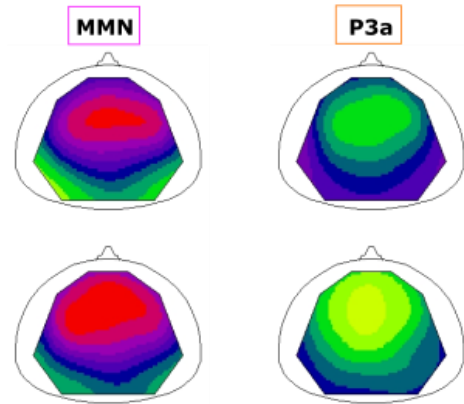

$+/-1.7$ uV

$+/-1.7$ uV

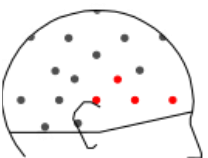

Right Fp

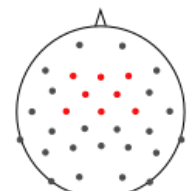

AMUSICS

FC

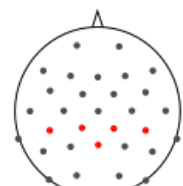

$\mathrm{CP}$

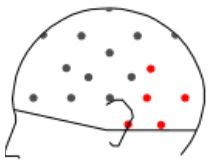

CONTROLS
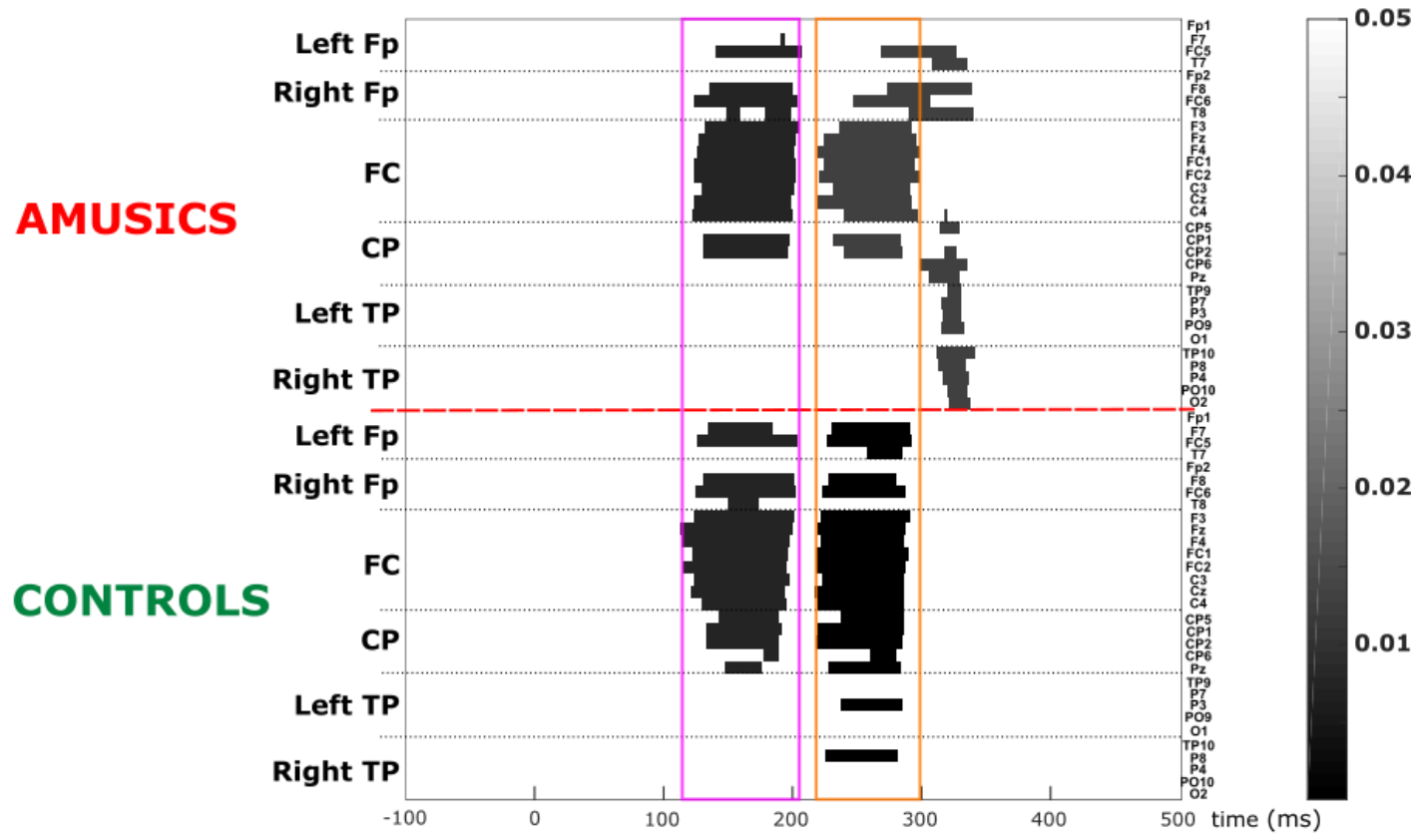

Left TP
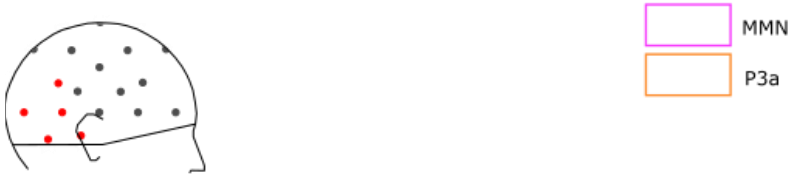

Right TP

Figure 3: Evoked response to an emotional anger deviant in Amusics and Controls. A) Average curve of eight fronto-central electrodes (Fp1, Fp2, F3, F4, FC1, FC2, C3, C4) of the response to the anger deviant minus the response to the neutral standard, for amusics and controls, negativity is up. B) Topographies for the two evoked potentials (MMN and P3) over the emergence windows identified below, separately for amusics and controls. Amplitude scale is indicated for each ERP. C) Emergence of evoked responses for amusics and controls for each electrode, grouped by topography, emergence windows used for the analysis are in pink for the MMN (113-205ms), orange for the P3a (217-299ms). Fp= pre-frontal, FC=fronto-central, $\mathrm{CP}=$ centro-parietal, TP=temporo-parietal. 


\begin{tabular}{|c|c|c|c|}
\hline & Amusics $(n=19)$ & Controls $(n=21)$ & $\begin{array}{l}\text { p-value (group } \\
\text { comparison) }\end{array}$ \\
\hline \multirow[t]{3}{*}{ Age (years) } & $30.7( \pm 14.38)$ & $32.33( \pm 14.5)$ & 0.72 \\
\hline & Min: 18 & Min: 19 & \\
\hline & Max: 56 & Max: 64 & \\
\hline \multirow[t]{3}{*}{ Education (years) } & $15( \pm 2.67)$ & $15.23( \pm 2.19)$ & 0.76 \\
\hline & Min: 10 & Min: 12 & \\
\hline & Max: 20 & Max: 20 & \\
\hline \multirow{3}{*}{$\begin{array}{l}\text { Musical training } \\
\text { (years) }\end{array}$} & 0 & $0.048( \pm 0.22)$ & 0.33 \\
\hline & & Min: 0 & \\
\hline & & Max: 1 & \\
\hline Sex & $9 \mathrm{M} 10 \mathrm{~F}$ & $8 \mathrm{M} 13 \mathrm{~F}$ & 0.55 \\
\hline Handedness & $5 \mathrm{~L} 14 \mathrm{R}$ & 4L 17R & 0.58 \\
\hline \multirow[t]{3}{*}{ MBEA score } & $22.02( \pm 1.8)$ & $26.45( \pm 1.04)$ & $<0.001$ \\
\hline & Min: 16.83 & Min: 24.8 & \\
\hline & Max: 24.5 & Max: 28.5 & \\
\hline \multirow[t]{3}{*}{ MBEA pitch score } & $21.05( \pm 1.97)$ & $26.6( \pm 1.42)$ & $<0.001$ \\
\hline & Min: 15.67 & Min: 23.33 & \\
\hline & Max: 23.67 & Max: 28.67 & \\
\hline \multirow[t]{3}{*}{ PDT (semitones) } & $1.33( \pm 1.48)$ & $0.29( \pm 0.15)$ & 0.007 \\
\hline & Min: 0.11 & Min: 0.08 & \\
\hline & Max: 4.99 & Max: 0.71 & \\
\hline
\end{tabular}


849 Table 1: Characteristics of the participants in both groups. The MBEA (Montreal Battery for the 850 Evaluation of Amusia, Peretz et al., 2003) score corresponds to the average of the six subtests of the 851 battery (maximum score $=30$, cut off: 23 ). Pitch mean score corresponds to the average of the three 852 pitch subtests in the MBEA (scale, contour and interval, cut off: 22). Note that a participant was 853 considered as amusic if any of these two measures (MBEA score, MBEA pitch score) was below the 854 cut-off. PDT: Pitch Discrimination Threshold (see Tillmann et al., 2009). For each variable (except sex 855 and handedness), the mean value in each group is reported along with the standard deviation in 856 parentheses. Groups were compared with t.tests (two sided), except for sex and handedness where a 857 Chi2 test was used (Qobs=0.35 and Qobs=0.3, respectively). 
bioRxiv preprint doi: https://doi org/10.1101/2020.08.05.238204; this version posted August 5,2020 . The copyright holder for this preprint (which was not certified by peer review) is the author/funder, who has granted bioRxiv a license to display the preprint in perpetuity. It is made available under aCC-BY-NC-ND 4.0 International license.

\begin{tabular}{|c|c|c|c|c|}
\hline $\begin{array}{l}\text { Acoustic } \\
\text { parameters }\end{array}$ & Neutral standard & Neutral deviant & Sadness deviant & Anger deviant \\
\hline Pitch mean $(H z)$ & 241 & 199 & 228 & 278 \\
\hline $\begin{array}{l}\text { Spectral flux mean } \\
\text { (a.u.) }\end{array}$ & 17.19 & 9.33 & 25.75 & 68.70 \\
\hline $\begin{array}{l}\text { Brightness mean } \\
\text { (a.u.) }\end{array}$ & 0.20 & 0.13 & 0.23 & 0.27 \\
\hline $\begin{array}{l}\text { Roughness mean } \\
\text { (a.u.) }\end{array}$ & 22.98 & 38.09 & 14.21 & 114.80 \\
\hline $\begin{array}{l}\text { Inharmonicity } \\
\text { mean (a.u.) }\end{array}$ & 0.18 & 0.17 & 0.27 & 0.45 \\
\hline Attack time (s) & 0.028 & 0.039 & 0.056 & 0.13 \\
\hline
\end{tabular}

\section{Behavioral data}

(Pralus et al.,

2019)

\section{$\%$ Correct}

recognition in

\section{Controls}

\% Correct

recognition in

Amusics
100

83

94

72

94

83

56

67 
bioRxiv preprint doi: https://doi.org/10.1101/2020.08.05.238204; this version posted August $5,2020$. The copyright holder for this preprint (which was not certified by peer review) is the author/funder, who has granted bioRxiv a license to display the preprint in perpetuity. It is made available under aCC-BY-NC-ND 4.0 International license.

\begin{tabular}{|c|c|c|c|c|}
\hline $\begin{array}{l}\text { Mean Intensity } \\
\text { ratings in Contro }\end{array}$ & NA & NA & 2.8 & 2.6 \\
\hline $\begin{array}{l}\text { Mean Intensity } \\
\text { ratings in Amusic }\end{array}$ & NA & NA & 2.8 & 2.4 \\
\hline
\end{tabular}

859 Table 2: Acoustic parameters of the stimuli and associated behavioral data from Pralus et al., 860 (2019). The acoustic parameters were computed with the MIR Toolbox (Lartillot \& Toiviainen, 2007), 861 with a temporal frame of 50ms. a.u.: arbitrary units. Percentage of correct emotion recognition and 862 intensity ratings (on a scale from 1 to 5) for these stimuli are from Pralus et al. (2019) and were obtained 863 from 18 congenital amusics and 18 matched controls. NA: not applicable, no intensity ratings was given 864 for neutral stimuli. 